\title{
Bid Rotation and Collusion in Repeated Auctions
}

\author{
Masaki Aoyagi
}

University of Pittsburg

May 2000

\begin{abstract}
This paper studies bidder collusion with communication in repeated auctions when no side transfer is possible. It presents a simple dynamic bid rotation scheme with co-ordinates bids based on communication history and enables intertemporal transfer of bidders' payoffs. The paper derives a sufficient condition for such a dynamic scheme to be an equilibrium and characterises the equilibrium payoffs in a general environment with affiliated signals and private or interdependent values. With IPV, it is shown that this dynamic scheme yields a strictly higher payoff to the bidders than any static collusion scheme which co-ordinates bids based only on the current reported signals.
\end{abstract}

\section{Acknowledgements}

I am grateful to Uday Rajan and Phil Reny for helpful comments. Financial support from the Faculty of Arts and Sciences of the University of Pittsburg is gratefully acknowledged.

\author{
Address for Correspondence \\ Department of Economics \\ University of Pittsburg \\ Pittsburg \\ PA 15260 \\ USA \\ Maoyagi+@pitt.edu
}




\section{Introduction}

It is well recognized that bidder collusion is a serious problem in many auctions: Collusion is documented in auctions for used machinery, timbers, frequency spectrums, Treasury securities, the procurement of construction work, etc. (Marshall and Meurer (1995), Porter and Zona (1990), Baldwin et al. (1997)). Despite its significance as an empirical phenomenon, relatively little is understood about the theory of collusion in auctions, which is distinguished from the standard collusion theory by the presence of asymmetric information across bidders about their valuations of the object.

Most of the existing analysis of collusion in auctions is conducted in the one-shot framework. One important contribution in this case is made by McAfee and McMillan (1992), who analyze bidder collusion with communication in first-price auctions under the independent private values (IPV) assumption. Their key findings include the identification of the most efficient collusion schemes with and without side transfer. In particular, they show that full collusion is possible with side transfer, but that the scope of bidder collusion is severely limited without it.

If collusion is a product of frequent interaction, however, a more appropriate framework for analysis is that of repeated games, where the same set of bidders participate in a series of auctions held sequentially over time. ${ }^{1}$ The purpose of this paper is to show that in infinitely repeated auctions, collusion is possible through intertemporal payoff transfer even if there is no side payment of money. In other words, bidders in repeated auctions can collude through the adjustment of continuation payoffs in a way that partially compensates for the lack of monetary transfer. The paper derives a sufficient condition for such a collusion scheme to be an equilibrium and characterizes the equilibrium payoffs in a general environment with affiliated signals and private or interdependent values.

We consider a model of infinitely repeated auctions with two symmetric bidders. In every period, a single indivisible object is sold through the same auction format, and the bidders' private signals are drawn from the same distribution.

The bidders collude by coordinating their bids in each auction with the help of a media-

\footnotetext{
${ }^{1}$ See also Hendricks and Porter (1990), who emphasize the need of a repeated model of collusion in auctions.
} 
tion device referred to here as a center. In each period, the center receives reports from the bidders about their private signals and then instructs them on what bid to submit in the stage auction. This stage mechanism, which chooses instructions as a function of reports, is called an instruction rule in this paper. A collusion scheme represents the center's choice of an instruction rule in every period contingent on history. A collusion scheme is an equilibrium if truth-telling is incentive compatible and obedience to the instructions is rational for the bidders.

This paper examines a bid rotation scheme which instructs no more than one bidder to participate in each stage auction during the collusion phase, and punishes any deviation from the instruction by reversion to the one-shot Nash equilibrium of the stage auction. In particular, we will construct a bid rotation scheme which uses three different instruction rules during the collusion phase as follows: In the original symmetric phase $S$, the center uses the efficient instruction rule which instructs the bidder with the higher valuation (based on the reports) to bid the reservation price $R$ if and only if his valuation exceeds $R$. It instructs the other bidder to stay out. In phase $A_{i}(i=1,2)$, the center uses an asymmetric instruction rule which disfavors bidder $i$ in the sense that $i$ 's $e x$ ante payoff is lower than that of bidder $j$.

It can be seen that the efficient instruction rule used in phase $S$ is not incentive compatible since the bidders tend to overstate their signals in the hope of winning the object at the reservation price. We suppose that the incentive for truth-telling in phase $S$ is provided through the adjustment in continuation payoffs as follows: When bidder $i$ 's reported signal is higher than that of $j$, a transition to phase $A_{i}$ takes place with positive probability so that bidder $i$ 's continuation payoff would be lower. The instruction rule used in phase $A_{i}$ is chosen to be incentive compatible so that no further adjustment in continuation payoffs is necessary. Phase $A_{i}$ lasts for a fixed number of periods, and the game returns to phase $S$.

The above discussion suggests that the bid rotation scheme considered in this paper is a dynamic scheme which chooses a different instruction rule contingent on the communication history. On the other hand, the one-shot collusion scheme analyzed by McAfee and McMillan (1992) corresponds to a static bid rotation scheme in repeated auctions which uses the same instruction rule every period. Note in particular that any instruction rule used by a static 
collusion scheme must be incentive compatible since no adjustment in continuation payoffs is possible. The advantage of dynamic bid rotation is most evident in the IPV environment. Specifically, when the auction is first-price, it can be shown that the dynamic scheme is an equilibrium for sufficiently patient bidders and yields a strictly higher payoff to them than the optimal static scheme without side transfer. The intuition is as follows: The optimal static scheme of McAfee and McMillan (1992) uses a random instruction rule whose support consists of the two asymmetric instruction rules used in phases $A_{1}$ and $A_{2}$ of the dynamic scheme described above. It follows that the average of a bidder's payoffs in these two phases exactly equals his payoff from the optimal static scheme. Since the allocation in phase $S$ is efficient, the bidder's overall payoff in the repeated game is strictly higher.

It should be noted that the collusion scheme described above does not extract all the surplus of the trade from the auctioneer. In other words, the scheme is not first-best efficient from the point of view of the bidders. The question of first-best efficiency in collusion without side transfer under asymmetric information is indeed very difficult. Efficiency results are available only in IPV models with finite signals: Fudenberg et al. (1994) show that the IPV model with finite signals and communication has the "product structure," which guarantees the existence of a near efficient equilibrium for sufficiently low discounting. ${ }^{2}$ As is often the case with mechanism design problems, however, conclusions based on finite signals do not necessarily extend to continuous signals. ${ }^{3}$

As mentioned above, most existing models of collusion in auctions are one-shot. Robinson (1985) and von Ungern-Sternberg (1988) are among the first to point out the vulnerability of the English and second-price sealed-bid auctions to bidder collusion. ${ }^{4}$ Graham and Marshall (1987) and Graham et al. (1990) present particular side transfer schemes for second-price and English auctions with pre-auction communication. Subsequently, Mailath and Zemsky (1991) and McAfee and McMillan (1992) identify the optimal schemes under the IPV assumption.

\footnotetext{
${ }^{2}$ Based on this result, Athey and Bagwell (1999) characterize the first-best scheme in the IPV model with binary signals.

${ }^{3}$ The finiteness of actions is critical. With communication, a player's action includes a mapping from the set of signals to the set of reports. This suggests that using a finite message space in communication is not sufficient.

${ }^{4}$ Engelbrecht-Wiggans and Kahn (1998) and Brusco and Lopomo (2000) analyze collusion in (one-shot) multi-object ascending price auctions.
} 
The former examine collusion with side transfer in second-price auctions, while the latter look at that with and without side transfer in first-price auctions. Both papers conclude that efficient collusion is possible with side transfer. ${ }^{5}$ More recently, Athey et al. (1998), Johnson and Robert (1998), and Skrzypacz and Hopenhayn (1999) analyze collusion in repeated IPV auctions without side transfer. ${ }^{6}$ Among them, Skrzypacz and Hopenhayn (1999) prove the existence of a collusion scheme without communication that performs strictly better than the static scheme of McAfee and McMillan (1992) when the stage auction is first-price and the reservation price equals zero. While the intuition behind their results is closely related to ours, their formal logic is specialized to the particular auction format as well as the IPV assumption. In contrast, one of this paper's main objectives is to present a simple collusion scheme which is robust with respect to these specifications.

In this paper, it is assumed that every stage auction has the same format. While we believe that it is an appropriate description of many actual practices, an alternative formulation would include the auctioneer as an active player of the game. In the one-shot framework, Mailath and Zemsky (1991) and McAfee and McMillan (1992) both discuss the choice of the reservation price as the auctioneer's response to collusion. In repeated auctions, the corresponding treatment is to let the auctioneer choose the reservation price as a function of history. The analysis of such a model is left as a topic of future research.

The organization of the paper is as follows: The next section formulates a model of repeated auctions with the center as a mediation device. The dynamic bid rotation scheme is described in Section 3. The main theorem in this section gives a sufficient condition for this scheme to be an equilibrium and describes the equation that characterizes its payoff. Section 4 provides a comparison of the dynamic and static schemes under the IPV assumption. Section 5 discusses the generalization of the sufficient condition for the existence of an equilibrium dynamic bid rotation scheme that yields a strictly higher payoff than the optimal static scheme.

\section{Model}

There are two risk-neutral bidders 1 and 2 and a center which coordinates their bidding

${ }^{5}$ An earlier version of this paper contains an extension of this result.

${ }^{6}$ I became aware of these papers after the first version of the present paper was completed. 
in infinitely repeated auctions. A single indivisible object is sold every period through a fixed auction format. In each period, bidder $i$ receives a private signal $s_{i} \in[0,1]$ about the value of the object. The probability distribution of the signal profile $s=\left(s_{1}, s_{2}\right)$ is the same in every period and represented by the density function $f$ whose support is the unit square $[0,1]^{2}$. The signals are independent across periods. The conditional density of $s_{i}$ given $s_{j}$ is denoted $f_{i}\left(\cdot \mid s_{j}\right)$, and the corresponding distribution function is denoted $F_{i}\left(\cdot \mid s_{j}\right)(i \neq j)$. With slight abuse of notation, we also use $f_{i}$ (resp. $F_{i}$ ) to denote the marginal density (resp. distribution $)$ of $s_{i}(i=1,2)$. We assume that

Assumption 1: The signals $s=\left(s_{1}, s_{2}\right)$ are affiliated.

See Milgrom and Weber (1982) for the definition of affiliation as well as its properties. Assumption 1 is standard in auction theory and includes independent signals as a special case. Our analysis will only use the following property of affiliation (e.g., Milgrom and Weber (1982, Lemma 1)):

$$
\frac{f_{i}\left(s_{i} \mid s_{j}\right)}{F_{i}\left(s_{i} \mid s_{j}\right)} \text { is weakly increasing in } s_{j} \text { for any } s_{i}(i \neq j) .
$$

Given the signal profile $s=\left(s_{1}, s_{2}\right)$, the expected value of the object to bidder $i$ is denoted $v_{i}(s) \geq 0$. Throughout, we adopt the convention that the first argument of $v_{i}$ is $s_{i}$ (own signal) and the second is $s_{j}$ (the other bidder's signal). The value function $v_{i}$ is the same for every period.

Throughout, the problem is assumed to be symmetric in the sense that $f(\alpha, \beta)=f(\beta, \alpha)$ and $v_{i}(\alpha, \beta)=v_{j}(\alpha, \beta)$ for every $\alpha, \beta \in[0,1]$.

The following two possibilities will be considered concerning the functional form of $v_{i}$. The values are private if $v_{i}(s)=s_{i}$ for every $s=\left(s_{i}, s_{j}\right)$, and interdependent if $v_{i}$ is strictly increasing in both $s_{i}$ and $s_{j}(i=1,2, j \neq i)$. In the case of interdependent values, the function $v_{i}$ is assumed to satisfy the following regularity conditions.

Assumption 2: For $i=1,2$ and $j \neq i$,

(i) $v_{i}$ is continuously differentiable.

(ii) $\frac{\partial v_{i}}{\partial s_{i}}(\alpha, \alpha)>\frac{\partial v_{j}}{\partial s_{i}}(\alpha, \alpha)$ for every $\alpha \in[0,1]$.

Assumption 2(ii) is the single-crossing property commonly assumed in the auction literature. It should be noted that in a private values model, Assumption 2 is automatically 
satisfied. A stage auction in this paper is any transaction mechanism that solicits a single sealed bid from each bidder and then determines the allocation of the good as well as monetary transfer. ${ }^{7}$ Participation in the stage auction is voluntary so that the set of each bidder's generalized bids is expressed as $B=\{N\} \cup \mathbf{R}_{+}$, where $N$ represents "no participation." The rule of the auction is summarized by measurable mappings $\theta_{i}$ and $p_{i}(i=1,2)$ on the set $B^{2}$ of bid profiles $b=\left(b_{1}, b_{2}\right): \theta_{i}(b)$ is the probability that bidder $i$ is awarded the good, and $p_{i}(b)$ is his expected payment to the auctioneer. The functions $\theta_{i}$ and $p_{i}(i=1,2)$ are symmetric in the sense that $\theta_{i}(\alpha, \beta)=\theta_{j}(\alpha, \beta)$ and $p_{i}(\alpha, \beta)=p_{j}(\alpha, \beta)$ for any $\alpha, \beta \in[0,1]$, and satisfy the following conditions.

Assumption 3: (i) A bidder makes no payment when he chooses not to participate: $p_{i}(b)=$ 0 if $b_{i}=N$.

(ii) There exists a non-random reservation price $R\left(\in\left[0, v_{i}(1,1)\right)\right)$ such that a bidder may win the object only if he submits a bid at or above $R: \theta_{i}(b)=0$ if $b_{i} \in\{N\} \cup[0, R)$.

(iii) If only one bidder participates and submits bid $R$, then he wins the object at price $R$ : $\theta_{i}(b)=1$ and $p_{i}(b)=R$ if $b_{i}=R$ and $b_{j}=N$.

(iv) There exists a symmetric Nash equilibrium in the (Bayesian) game in which each bidder's strategy is a mapping $\eta_{i}:[0,1] \rightarrow B$ and payoff function is

$$
\int_{[0,1]^{2}}\left\{\theta_{i}(\eta(s)) v_{i}(s)-p_{i}(\eta(s))\right\} f(s) d s .
$$

Assumption 3 encompasses most standard auctions including the first- and second-price auctions. Let $g^{0}$ be the (ex ante) symmetric Nash equilibrium payoff to each bidder in the stage auction as described in Assumption 3(iv). Also, let $g^{*}$ be the expected payoff to each bidder under truthful information sharing and efficient allocation with bidder $i$ winning the object at price $R$ if and only if $v_{i}$ exceeds both $v_{j}$ and $R$ :

$$
g^{*}=\int_{\left\{s: v_{i}(s)>\max \left\{v_{j}(s), R\right\}\right\}}\left\{v_{i}(s)-R\right\} f(s) d s .
$$

Clearly, $2 g^{*}$ gives the first-best joint collusive payoff, and the bidders see a potential gain from collusion if $g^{0}<g^{*}$. This is the case to be studied in what follows.

\footnotetext{
${ }^{7}$ The restriction to a seal-bid auction is purely for simplicity.
} 
Collusion in the repeated auction takes the following form: At the beginning of each period, the two bidders report their private signals $s_{i}$ to the center. Upon receiving the report profile $\hat{s}=\left(\hat{s}_{1}, \hat{s}_{2}\right) \in[0,1]^{2}$, the center chooses instruction to each bidder $i$ on what (generalized) bid to submit in the stage auction.

In general, the bidders may report a false signal, and/or disobey the instruction. Bidder $i$ 's reporting rule $\lambda_{i}:[0,1] \rightarrow[0,1]$ chooses report $\hat{s}_{i}$ as a function of signal $s_{i}$, and his bidding rule $\mu_{i}:[0,1]^{2} \times B \rightarrow B$ chooses bid $b_{i}$ in the stage action as a function of his signal, report and instruction. The reporting rule is honest if it always reports the true signal, and the bidding rule is obedient if it always obeys the instruction. Denote by $\lambda_{i}^{*}$ and $\mu_{i}^{*}$ bidder $i$ 's honest reporting rule and obedient action rule, respectively.

For simplicity, we assume that the (generalized) bids in the stage auction are observable to every party including the center. ${ }^{8}$ It should be noted that the conclusion of this paper continues to hold in an alternative framework where only the identity of the winner is publicly observable. This is because (i) the designated winner in each stage auction obtains the object at the lowest price possible (= reservation price) in the collusion scheme considered in this paper, and (ii) the designated loser may gain from disobeying the instruction only by changing the identity of the winner. The observability of bids implies that a bidder's deviation can be classified into two types: A bidder commits an observable deviation when he chooses a bid different from the instruction given to him, and commits an unobservable deviation when he reports a false signal.

The center is formally a communication device as defined by Forges (1986) and Myerson (1986). Its choice of instructions to the bidders given their reports is captured by an instruction rule $q=\left(q_{1}, q_{2}\right):[0,1]^{2} \rightarrow B^{2}: q_{i}(\hat{s})$ is the instruction to bidder $i$ when the report profile is $\hat{s}$. Let $g_{i}(\lambda, \mu, q)$ denote bidder $i$ 's stage payoff resulting from any profile $(\lambda, \mu, q)$ of reporting and bidding rules $(\lambda, \mu)=\left(\lambda_{1}, \mu_{1}, \lambda_{2}, \mu_{2}\right)$ and instruction rule $q$. The instruction rule $q$ is one-shot incentive compatible (one-shot IC) if neither bidder has an incentive to misreport his signal: $g_{i}\left(\lambda^{*}, \mu^{*}, q\right) \geq g_{i}\left(\lambda_{i}, \lambda_{j}^{*}, \mu^{*}, q\right)$ for any reporting rule $\lambda_{i}, i=1,2$, and $j \neq i$. Note that one-shot incentive compatibility refers only to the incentive in reporting

${ }^{8}$ If the center is a simple communication device which does not have any observation function of its own, we can let the bidders report each other's bid to the center so that its instructions in the next period will be conditional on the reports. 
and presumes bidders' obedience to the given instructions. In particular, the instruction rule that instructs bidders to play the one-shot Nash equilibrium of the stage auction is one-shot IC. Lemma 1 in the next section identifies some other instruction rules with this property.

Bidder i's communication history in period $t$ in the repeated auction game is the sequence of his reports and instructions in periods $1, \ldots, t-1$. On the other hand, bidder i's private history in period $t$ is the sequence of his private signals $s_{i}$ in periods $1, \ldots, t-1$. Furthermore, the public history in period $t$ is a sequence of instruction rules used by the center in periods $1, \ldots, t$ and (generalized) bid profiles in the stage auctions in periods $1, \ldots, t-1$.

Bidder $i$ 's (pure) strategy $\sigma_{i}$ in the repeated auction chooses the pair $\left(\lambda_{i}, \mu_{i}\right)$ of reporting and bidding rules in each period $t$ as a function of his communication and private histories in $t$, and the public history in $t$. Let $\sigma_{i}^{*}$ be bidder $i$ 's honest and obedient strategy which plays the pair $\left(\lambda_{i}^{*}, \mu_{i}^{*}\right)$ of the honest reporting rule and obedient bidding rule for all histories.

The collusion scheme $\tau$ describes the center's choice of an instruction rule in every period as a function of communication and public histories. At the beginning of each period, it publicly informs the bidders which instruction rule is used in that period.

Our analysis will focus on the following class of "grim-trigger" collusion schemes with two phases: The game starts in the collusion phase, and reverts to the punishment phase forever if and only if there is an observable deviation by either bidder in the sense described above. In the punishment phase, the bidders are instructed to play the one-shot Nash equilibrium of the stage auction specified in Assumption 3(iv).

A collusion scheme $\tau$ in this class is static if it chooses the same instruction rule in every period during the collusion phase independent of the history, and is dynamic otherwise. Furthermore, the collusion scheme $\tau$ employs bid rotation if no more than one bidder is instructed to participate in each stage auction during the collusion phase.

Let $\delta<1$ be the bidders' common discount factor, and $\Pi_{i}(\sigma, \tau)$ be bidder $i$ 's average discounted payoff (normalized by $(1-\delta)$ ) in the repeated game under the strategy profile $(\sigma, \tau)$. The collusion scheme $\tau$ is an equilibrium if the pair $\sigma^{*}=\left(\sigma_{1}^{*}, \sigma_{2}^{*}\right)$ of honest and obedient strategies constitutes a Nash equilibrium of the repeated game: $\Pi_{i}\left(\sigma^{*}, \tau\right) \geq \Pi_{i}\left(\sigma_{i}^{\prime}, \sigma_{j}^{*}, \tau\right)$ for any $\sigma_{i}^{\prime}, i=1,2$, and $j \neq i$. It follows from the definition that if $\tau$ is an equilibrium static collusion scheme, then its instruction rule in the collusion phase is one-shot IC. 


\section{A Dynamic Bid Rotation Scheme}

Let $q^{*}$ be the efficient instruction rule that instructs bidder $i$ to bid $R$ if his valuation $v_{i}(\hat{s})$ (based on the report profile $\hat{s}$ ) is higher than both $R$ and $v_{j}(\hat{s})$, and to stay out otherwise:

$$
q_{i}^{*}(\hat{s})= \begin{cases}R & \text { if } v_{i}(\hat{s})>\max \left\{R, v_{j}(\hat{s})\right\} \\ N & \text { otherwise. }\end{cases}
$$

Clearly, the (ex ante) payoff $g_{i}\left(\lambda^{*}, \mu^{*}, q^{*}\right)$ associated with $q^{*}$ equals the first-best level $g^{*}$ although $q^{*}$ is not one-shot IC.

Consider next the asymmetric instruction rule $q^{i}$ that $(i)$ instructs bidder $j(\neq i)$ to bid $R$ if his valuation $v_{j}(\hat{s})$ exceeds $R$, and to stay out otherwise, and (ii) instructs bidder $i$ to bid $R$ if his valuation $v_{i}(\hat{s})$ exceeds $R$ and if bidder $j$ 's valuation $v_{j}$ would not exceed $R$ even when $i$ 's signal were 1 :

$$
q_{i}^{i}(\hat{s})=\left\{\begin{array}{ll}
R & \text { if } v_{i}(\hat{s})>R \geq v_{j}\left(\hat{s}_{j}, 1\right), \\
N & \text { otherwise }
\end{array} \quad q_{j}^{i}(\hat{s})= \begin{cases}R & \text { if } v_{j}(\hat{s})>R \\
N & \text { otherwise }\end{cases}\right.
$$

In other words, $q^{i}$ treats bidders $j$ and $i$ as the primary and secondary bidders, respectively. Let $\underline{g}=g_{i}\left(\lambda^{*}, \mu^{*}, q^{i}\right)$ and $\bar{g}=g_{j}\left(\lambda^{*}, \mu^{*}, q^{i}\right)$ be the bidders' expected payoffs under $q^{i}$. By definition, $\bar{g}>\underline{g}$.

LEMMA 1. $q^{i}$ is one-shot IC.

Proof: See the Appendix.

Since $2 g^{*}$ is the first-best joint collusive payoff, it can be readily verified that

$$
2 g^{*}>\bar{g}+\underline{g},
$$

where the strict inequality is the consequence of the full support of the density function $f$ and $R<v_{i}(1,1)$. Let $\tau^{d}$ be a dynamic bid rotation scheme such that:

a) The collusion phase consists of three subphases $S, A_{1}$ and $A_{2}: S$ is the original symmetric phase where the efficient instruction rule $q^{*}$ is used, while $A_{i}$ is the asymmetric phase where the instruction rule $q^{i}$ is used.

b) Play begins in the symmetric phase $S$. After each period in phase $S$, transition to the asymmetric phase $A_{i}(i=1,2)$ takes place with probability $\omega_{i}(\hat{s})$, which is a function of the 
reported signals in the current period alone and given by

$$
\omega_{i}(\hat{s})= \begin{cases}x\left(\hat{s}_{i}\right) & \text { if } \hat{s}_{i}>\hat{s}_{j} \\ 0 & \text { otherwise }\end{cases}
$$

for some increasing function $x:[0,1] \rightarrow[0,1]$. Play stays in phase $S$ with probability $1-\omega_{1}(\hat{s})-\omega_{2}(\hat{s})$.

c) Each asymmetric phase $A_{i}$ lasts exactly for $m$ periods and then play returns to $S$.

It should be noted that the transition probability $x\left(\hat{s}_{i}\right)$ depends only on the higher of the two reports. There is a clear connection between the above collusion scheme and the one with side transfer in McAfee and McMillan (1992). In McAfee and McMillan (1992), each bidder is discouraged from overstating his signal by the transfer payment that is required from the bidder with the higher report. On the other hand, the deterrent in the above scheme is the possibility of a lower continuation payoff for such a bidder. This is a natural modification of the side transfer scheme in view of the substitutability of continuation payoffs for monetary transfer in repeated games.

Let $r \in[0,1]$ be the (unique) signal such that $v_{i}(r, r)=R$, and define the function $y:(\underline{g}, \infty) \rightarrow \mathbf{R}_{++}$by

$$
y(u)=\int_{r}^{1} \int_{0}^{s_{i}} \int_{r}^{s_{i}}\left\{v_{i}(\beta, \beta)-R\right\} z_{i}(\beta) e^{-\frac{\bar{g}-\underline{g}}{u-\underline{g}} \int_{\beta}^{s_{i}} z_{i}(\gamma) d \gamma} d \beta f(s) d s_{j} d s_{i},
$$

where

$$
z_{i}(\beta)=\frac{f_{i}(\beta \mid \beta)}{F_{i}(\beta \mid \beta)}
$$

A bidder's payoff $u^{d}=\Pi_{i}\left(\sigma^{*}, \tau^{d}\right)$ from the dynamic bid rotation scheme $\tau^{d}$ is characterized by (i) the recursive equation on $u^{d}$ involving the transition probability function $x$ (in (b) above), and (ii) the incentive compatibility condition on $x$ for truthful reporting in phase $S$. By the standard argument, the latter gives rise to a linear differential equation of $x$ which has the payoff $u^{d}$ as a parameter. By substituting the solution to this equation into the recursive equation in (i), we obtain the following equation of $u=u^{d}$ :

$$
\varphi(u) \equiv u-g^{*}+\frac{2 u-\bar{g}-\underline{g}}{u-\underline{g}} y(u)=0 .
$$

Note that the function $\varphi:(\underline{g}, \infty) \rightarrow \mathbf{R}$ is continuous since $y$ is. The following is our main theorem. 
THEOREM 1. Assume that the values are either private or interdependent. If $\varphi(u)=0$ has a solution $u^{d}$ strictly greater than $g^{0}$, then for a sufficiently large discount factor $\delta$, the dynamic bid rotation scheme $\tau^{d}$ is an equilibrium for some $x$ (transition probability) and $m$ (duration of phase $A_{i}$ ), and yields payoff $u^{d}$.

Proof: See the Apendix.

It should be noted that low discounting is required for $\tau^{d}$ to be an equilibrium although (3) and its solution $u^{d}$ are independent of $\delta$. While $\varphi(u)=0$ is not analytically solvable in general, there is a simple sufficient condition based on the comparison of $g^{0}$ and $(\bar{g}+\underline{g}) / 2$ as follows: Since $y(u)>0$ for any $u>\underline{g}$, it readily follows from (2) that

$$
\varphi\left(g^{*}\right)=\frac{2 g^{*}-\bar{g}-\underline{g}}{g^{*}-\underline{g}} y\left(g^{*}\right)>0 \quad \text { and } \quad \varphi\left(\frac{\bar{g}+\underline{g}}{2}\right)=\frac{\bar{g}+\underline{g}}{2}-g^{*}<0 .
$$

By the intermediate value theorem, hence, there exists $u^{d} \in\left((\bar{g}+\underline{g}) / 2, g^{*}\right)$ that solves $\varphi(u)=0$. The intuition behind $u^{d}>(\bar{g}+\underline{g}) / 2$ is as follows: Phases $A_{1}$ and $A_{2}$ are equally likely $e x$ ante so that a bidder's expected payoff conditional on being in these two phases equals $(\underline{g}+\bar{g}) / 2$. Since allocation is efficient in phase $S$, his overall payoff in the repeated game exceeds it. This observation combined with Theorem 1 yields the following corollary.

Corollary 1. Assume that the values are either private or interdependent. If $g^{0} \leq(\bar{g}+$ $\underline{g}) / 2$, then for a sufficiently large discount factor $\delta$, the dynamic bid rotation scheme $\tau^{d}$ is an equilibrium for some $x$ and $m$.

EXAmPle 1: Suppose that the stage auction is second-price sealed-bid with the reservation price $R$ equal to zero. In this case, it is well known that the bidding function in the one-shot Nash equilibrium is given by $\eta_{i}^{0}\left(s_{i}\right)=v_{i}\left(s_{i}, s_{i}\right)$. Suppose further that the value function $v_{i}$ has the linear form $v_{i}(s)=c s_{i}+(1-c) s_{j}$, where $c \in(1 / 2,1]$. As seen, the values are private if $c=1$ and interdependent otherwise. The one-shot Nash equilibrium payoff equals

$$
g^{0}=\int_{\left\{s: s_{i}>s_{j}\right\}}\left\{v_{i}(s)-\eta_{j}^{0}\left(s_{j}\right)\right\} f(s) d s=c \int_{\left\{s: s_{i}>s_{j}\right\}}\left(s_{i}-s_{j}\right) f(s) d s,
$$

whereas $\underline{g}=0$ and

$$
\bar{g}=\int_{[0,1]^{2}}\left\{c s_{i}+(1-c) s_{j}\right\} f(s) d s .
$$


From these, we can verify that

$$
\frac{1}{2}(\underline{g}+\bar{g})-g^{0}=\frac{1}{2} \int_{[0,1]^{2}}\left\{s_{j}-c\left|s_{j}-s_{i}\right|\right\} f(s) d s .
$$

Therefore, the sufficient condition in Corollary 1 is equivalent to $E\left[s_{j}\right] \geq c E\left[\left|s_{j}-s_{i}\right|\right]$. It follows that for the fixed distribution $f$, if $\tau^{d}$ is an equilibrium in the private values model $(c=$ $1)$, then it is an equilibrium in the interdependent values model $(c<1)$ as well. Furthermore, since $E\left[s_{j}\right]>\frac{1}{2} E\left[\left|s_{i}-s_{j}\right|\right]$ for any $f$ as can be readily verified, there exists $\bar{c}>1 / 2$ such that if $c \leq \bar{c}$, then $\tau^{d}$ is an equilibrium.

\section{Dynamic vs. Static Collusion Schemes with IPV}

In this section, we apply Theorem 1 to the IPV environment where the signals are independent and the values are private. Theorem 2 below states that the condition of Theorem 1 holds when the stage auction is either first-price or second-price.

Theorem 2. Assume independent private values (IPV). Suppose that the stage auction is either first-price or second-price sealed-bid. Then for a sufficiently large discount factor $\delta$, the dynamic bid rotation scheme $\tau^{d}$ is an equilibrium for some $x$ and $m$, and its payoff $u^{d}$ is strictly higher than both $(\bar{g}+\underline{g}) / 2$ and $g^{0}$.

Proof: See the Apendix.

Based on the conclusion of Theorem 2, we now turn to the comparison of the collusive payoffs associated with static and dynamic collusion schemes under IPV. In first-price sealed-bid auctions with IPV, McAfee and McMillan (1992) show that a certain degree of collusion is possible in some cases even if there is no side transfer and the auction is oneshot. Their theorem directly applies to the current repeated game framework and yields the characterization of the most efficient equilibrium static collusion scheme. Let

$$
h_{i}\left(s_{i}\right)=\frac{1-F_{i}\left(s_{i}\right)}{f_{i}\left(s_{i}\right)}
$$

be the inverse hazard rate of $s_{i}$. The following theorem is stated without a proof as it is a straightforward application of Theorem 1 of McAfee and McMillan (1992).

Theorem 3. Assume independent private values (IPV). Suppose that the stage auction is first-price sealed-bid. Let $\tau^{s}$ be the most efficient equilibrium static collusion scheme without 
side transfer for a sufficiently large discount factor $\delta$. Then $\tau^{s}$ is a grim-trigger scheme, and its payoff $u^{s}$ is as follows:

(i) If $h_{i}(\cdot)$ is (weakly) increasing, then $u^{s}$ equals the one-shot equilibrium payoff $g^{0}$.

(ii) If $h_{i}(\cdot)$ is (weakly) decreasing, then $u^{s}$ equals $(\bar{g}+\underline{g}) / 2$.

Note that the static collusion scheme must use a one-shot IC instruction rule in every period since no incentive for truthful reporting can be provided through the adjustment in continuation payoffs. In particular, McAfee and McMillan (1992) show that when $h_{i}(\cdot)$ is decreasing (case (ii) above), the efficient static scheme $\tau^{s}$ uses a random instruction rule $\tilde{q}:[0,1]^{2} \rightarrow \Delta B^{2}$ which places probability one-half each on $q^{i}$ and $q^{j}$ defined in the previous section: $\tilde{q}=\frac{1}{2} q^{i}+\frac{1}{2} q^{j}$. More explicitly, $\tilde{q}$ can be written as

$$
\left(\tilde{q}_{i}(\hat{s}), \tilde{q}_{j}(\hat{s})\right)= \begin{cases}(R, N) & \text { if } \hat{s}_{i}>R \geq \hat{s}_{j}, \\ \frac{1}{2}(N, R)+\frac{1}{2}(R, N) & \text { if } \hat{s}_{i}, \hat{s}_{j}>R, \\ (N, N) & \text { if } \hat{s}_{i}, \hat{s}_{j} \leq R .\end{cases}
$$

In other words, the participating bidder is chosen at random with probability one-half when both valuations exceed $R$. Clearly, such an instruction rule is one-shot IC.

Comparison of Theorems 2 and 3 immediately reveals that when $h_{i}(\cdot)$ is monotone, the dynamic bid rotation scheme $\tau^{d}$ outperforms any static bid rotation scheme. The following corollary summarizes this observation.

Corollary 2. Assume independent private values (IPV). Suppose that the stage auction is first-price sealed-bid, and that $h_{i}(\cdot)$ is monotone. Then for a sufficiently large discount factor $\delta$, the dynamic bid rotation scheme $\tau^{d}$ is an equilibrium for some $x$ and $m$, and yields a strictly higher payoff than any equilibrium static bid rotation scheme.

EXAMPLE 2: Suppose that the stage-auction is first-price, and that $s_{i}$ has the uniform distribution over $[0,1]$. Assume that $\delta$ is sufficiently large. ${ }^{9}$ Since $h_{i}^{\prime}\left(s_{i}\right)<0$ for every $s_{i}$, the best static scheme yields $u^{s}=(\underline{g}+\bar{g}) / 2$ by Theorem 3. Table 1 below presents the values of $u^{d}, u^{s}$ and the one-shot equilibrium payoff $g^{0}$ as fractions of the first-best efficiency level $g^{*}$ for various values of $R$. It can be seen that $\tau^{d}$ extracts at least close to $90 \%$ of the surplus.

${ }^{9}$ When $R=0$, for example, $\tau^{d}$ is an equilibrium if $\delta \geq .95$. 


$\begin{array}{ccccc}R & g^{*} & \frac{u^{d}}{g^{*}} & \frac{u^{s}}{g^{*}} & \frac{g^{0}}{g^{*}} \\ .0 & .3333 & .886 & .750 & .500 \\ .1 & .2835 & .899 & .786 & .571 \\ .2 & .2347 & .912 & .818 & .636 \\ .3 & .1878 & .926 & .848 & .696 \\ .4 & .1440 & .938 & .875 & .750 \\ .5 & .1042 & .949 & .900 & .799 \\ .6 & .0693 & .961 & .923 & .847 \\ .7 & .0405 & .981 & .944 & .889\end{array}$

Table 1

\section{Sufficient Condition}

In this section, we reformulate the sufficient condition for the existence of an equilibrium dynamic collusion scheme that achieves a strictly higher payoff than the optimal static scheme. Specifically, this condition is expressed in terms of the payoff vectors associated with asymmetric one-shot IC instruction rules.

Corollary 1 shows that the payoff from the dynamic scheme $\tau^{d}$ is strictly higher than the average of the payoffs associated with two asymmetric instruction rules $q^{1}$ and $q^{2}$ (used in phases $A_{1}$ and $A_{2}$, respectively). By the same logic, if we construct a dynamic bid rotation scheme using any other pair of asymmetric (one-shot IC) instuction rules, then a bidder's payoff in that scheme is strictly higher than the average of his payoffs from these two instruction rules. Formally, define $V$ to be the set of payoff vectors associated with one-shot IC instruction rules:

$$
V=\left\{\left(g_{1}\left(\lambda^{*}, \mu^{*}, q\right), g_{2}\left(\lambda^{*}, \mu^{*}, q\right)\right): q \text { is a one-shot IC instruction rule }\right\} .
$$

By the standard argument, the set $V$ is closed. Note also that the one-shot Nash equilibrium payoff vector $\left(g^{0}, g^{0}\right)$ belongs to $V$. Let $u^{s}=\Pi_{i}\left(\sigma^{*}, \tau^{s}\right)$ be the payoff associated with the most efficient (symmetric) static collusion scheme $\tau^{s}$. Since $\tau^{s}$ uses a one-shot IC instruction rule as previously noted, it follows that $\left(u^{s}, u^{s}\right) \in V$. Graphically, $\left(u^{s}, u^{s}\right)$ is the right-most point of the intersection between $V$ and the 45 degree line through the origin. 
Theorem 4. Assume that the values are either private or interdependent. Suppose that the payoff vector $\left(u^{s}, u^{s}\right)$ associated with the most efficient symmetric static collusion scheme is not an extreme point of the set $V$. Then for a sufficiently large discount factor $\delta$, there exists an equilibrium dynamic bid rotation scheme $\tau^{d}$ whose payoff $u^{d}$ is strictly higher than $u^{s}$.

Proof: Since $\left(u^{s}, u^{s}\right)$ is not an extreme point, symmetry implies that there exist one-shot IC instruction rules $\hat{q}^{1}$ and $\hat{q}^{2}$ with the payoff vectors $\left(\underline{g}^{\prime}, \bar{g}^{\prime}\right) \in V$ and $\left(\bar{g}^{\prime}, \underline{g}^{\prime}\right) \in V$, respectively, for some $\underline{g}^{\prime}$ and $\bar{g}^{\prime}$ such that $\underline{g}^{\prime}<\bar{g}^{\prime}$ and $\left(\underline{g}^{\prime}+\bar{g}^{\prime}\right) / 2 \geq u^{s}$. We also have $2 g^{*}>\underline{g}^{\prime}+\bar{g}^{\prime}$ by the same logic as in (2). Modify $\tau^{d}$ in Section 3 so that it chooses $\hat{q}^{i}$ in subphase $A_{i}(i=1$, 2). By Corollary $1, \tau^{d}$ is an equilibrium for sufficiently patient bidders and its payoff $u^{d}$ is strictly higher than $\left(\bar{g}^{\prime}+\underline{g}^{\prime}\right) / 2 \geq u^{s}$. //

For example, suppose that the stage-auction is first-price. Suppose further that IPV holds and and that $h_{i}(\cdot)$ is decreasing. In this case, we know from Corollary 2 that the payoff vector $\left(u^{d}, u^{d}\right)$ associated with the dynamic bid rotation scheme $\tau^{d}$ strictly dominates $\left(u^{s}, u^{s}\right)$. In the light of Theorem 4 , this is precisely because $\left(u^{s}, u^{s}\right)$ is not an extreme point of $V:$ In fact, it equals the convex combination of $(\bar{g}, \underline{g})$ and $(\underline{g}, \bar{g}) \in V$ (Theorem 2 ).

To see that the condition of Theorem 4 is sufficient but not necessary, consider the firstprice IPV auctions with an increasing $h_{i}(\cdot)$. In this case, $\left(u^{s}, u^{s}\right)$ equals the one-shot Nash equilibrium payoff vector $\left(g^{0}, g^{0}\right)$ (Theorem 3$)$ and $\left(u^{d}, u^{d}\right)$ dominates $\left(g^{0}, g^{0}\right)$ regardless of whether it is an extreme point of $V$ or not (Theorem 2).

\section{Appendix}

The following notation is used for the discussion of the interdependant values case in the Appendix. For each $s_{i} \in[0,1]$, let $k_{i}\left(s_{i}\right)$ be the opponent's signal $s_{j}$ such that $v_{i}\left(s_{i}, s_{j}\right)=R$ if there exists any such $s_{j} \in[0,1]$. Let $k_{i}\left(s_{i}\right)=1$ if $v_{i}\left(s_{i}, s_{j}\right)<R$ for every $s_{j} \in[0,1]$, and $k_{i}\left(s_{i}\right)=0$ if $v_{i}\left(s_{i}, s_{j}\right)>R$ for every $s_{j} \in[0,1]$. It follows from the continuity and strict monotonicity of $v_{i}$ that $k_{i}$ is well-defined. Also, we have by definition

$$
v_{i}\left(s_{i}, k_{i}\left(s_{i}\right)\right) \begin{cases}\geq R & \text { if } k_{i}\left(s_{i}\right)=0 \\ =R & \text { if } k_{i}\left(s_{i}\right) \in(0,1), \\ \leq R & \text { if } k_{i}\left(s_{i}\right)=1\end{cases}
$$


Furthermore, since $v_{i}$ is continuously differentiable, $k_{i}\left(s_{i}\right)$ is continuously differentiable itself at any $s_{i}$ such that $k_{i}\left(s_{i}\right) \in(0,1)$ by the implicit function theorem. Figure 1 depicts $k_{i}$ for a generic (interdependent) value function $v_{i}$.

Proof of Lemma 1: The conclusion is straightforward if the values are private. We will show below that $q^{i}$ is one-shot IC for bidder $i$ in the interdependent values case. A similar argument proves the same for bidder $j$. Let $G_{i}^{i}\left(s_{i}, \hat{s}_{i}\right)$ be bidder $i$ 's interim stage payoff under $q^{i}$ when he has signal $s_{i}$ and reports $\hat{s}_{i}$.

Let $k_{i}$ be as defined above, and $w \in[0,1]$ denote the minimum of $s_{j}$ such that $v_{j}\left(s_{j}, 1\right) \geq R$ (Figure 1). Note first that if $\hat{s}_{i}$ is such that $k_{i}\left(\hat{s}_{i}\right) \geq w$, then $G_{i}^{i}\left(s_{i}, \hat{s}_{i}\right)=0$ for any $s_{i}$ since $v_{i}\left(\hat{s}_{i}, s_{j}\right) \geq R$ implies $s_{j} \geq k_{i}\left(\hat{s}_{i}\right) \geq w$ so that $v_{j}\left(s_{j}, 1\right) \geq R$. Suppose now that $k_{i}\left(\hat{s}_{i}\right)<w$. In this case, we have

$$
G_{i}^{i}\left(s_{i}, \hat{s}_{i}\right)=\int_{k_{i}\left(\hat{s}_{i}\right)}^{w}\left\{v_{i}(s)-R\right\} f_{j}\left(s_{j} \mid s_{i}\right) d s_{j} .
$$

Since $v_{i}\left(s_{i}, s_{j}\right) \geq R$ for any $s_{j} \geq k_{i}\left(s_{i}\right)$ and $v_{i}\left(s_{i}, s_{j}\right) \leq R$ for any $s_{j} \leq k_{i}\left(s_{i}\right)$, it follows from the above equation that $G_{i}^{i}\left(s_{i}, \hat{s}_{i}\right)$ is maximized when $\hat{s}_{i}=s_{i} \cdot / /$

Proof of Theorem 1: Let $u>g^{0}$ be a solution to (3). Take any $m \in \mathbf{N}$ such that

$$
m>\frac{1}{u-\underline{g}} \int_{r}^{1}\left\{v_{i}(\beta, \beta)-R\right\} z_{i}(\beta) d \beta .
$$

We claim that there exists $\underline{\delta}<1$ such that for any $\delta>\underline{\delta}$,

$$
(1-\delta)\left\{v_{i}(1,1)-R\right\}+\delta g^{0}<\left(1-\delta^{m}\right) \underline{g}+\delta^{m} u
$$

and

$$
\frac{1-\delta}{\delta\left(1-\delta^{m}\right)(u-\underline{g})} \int_{r}^{1}\left\{v_{i}(\beta, \beta)-R\right\} z_{i}(\beta) d \beta<1 .
$$

This is clear for (a1) since $u>g^{0}$ by assumption. For $m$ as specified, (a2) also holds since

$$
\lim _{\delta \rightarrow 1-} \frac{1-\delta}{\delta\left(1-\delta^{m}\right)}=\lim _{\delta \rightarrow 1-} \frac{1}{\delta\left(1+\delta+\cdots+\delta^{m-1}\right)}=\frac{1}{m}
$$

Recall that $x(\alpha)$ denotes the probability of transition to subphase $A_{i}$ when $i$ 's report $\alpha$ is higher than that of bidder $j$. Take any $\delta>\underline{\delta}$ and let $x$ be given by

$$
x(\alpha)= \begin{cases}\frac{1-\delta}{\delta\left(1-\delta^{m}\right)(u-\underline{g})} \int_{r}^{\alpha}\left\{v_{i}(\beta, \beta)-R\right\} z_{i}(\beta) e^{-\frac{\bar{g}-\underline{g}}{u-\underline{g}} \int_{\beta}^{\alpha} z_{i}(\gamma) d \gamma} d \beta & \text { if } \alpha>r, \\ 0 & \text { otherwise. }\end{cases}
$$


Note that $x(\alpha) \in[0,1]$ by our choice of $m$ and $\delta$ so that it is indeed a probability.

In what follows, we fix $u, m, \delta>\underline{\delta}, x$ as above and prove the theorem in three steps: Step 1 shows that each bidder's payoff is $u$ when they play the honest and obedient strategy $\sigma_{i}^{*}$ under $\tau^{d}$. Steps 2 and 3 then prove the non-profitability of observable and unobservable deviations, respectively. By the principle of optimality in dynamic programming, the latter two steps can be accomplished by checking the profitability of one-step deviations.

Step 1. $\Pi_{i}\left(\sigma^{*}, \tau^{d}\right)=u$.

Note that $u$ is the payoff associated with $\tau^{d}$ if and only if it satisfies the following recursive equation:

$$
\begin{aligned}
u=(1-\delta) g^{*} & +\delta\left[\int_{0}^{1} \int_{0}^{s_{i}}\left[x\left(s_{i}\right)\left\{\left(1-\delta^{m}\right) \underline{g}+\delta^{m} u\right\}+\left\{1-x\left(s_{i}\right)\right\} u\right] f(s) d s_{j} d s_{i}\right. \\
& \left.+\int_{0}^{1} \int_{s_{i}}^{1}\left[x\left(s_{j}\right)\left\{\left(1-\delta^{m}\right) \bar{g}+\delta^{m} u\right\}+\left\{1-x\left(s_{j}\right)\right\} u\right] f(s) d s_{j} d s_{i}\right]
\end{aligned}
$$

Using symmetry, we can rewrite (a4) as

$$
u=g^{*}-\frac{\delta\left(1-\delta^{m}\right)}{1-\delta}(2 u-\bar{g}-\underline{g}) \int_{r}^{1} \int_{0}^{s_{i}} x\left(s_{i}\right) f(s) d s_{j} d s_{i} .
$$

Substitution of (a3) into (a5) shows that the above recursive equation is equivalent to (3). Since $u$ solves (3) by assumption, the desired conclusion follows.

Step 2. No observable deviation (in bidding) is profitable.

When bidder $i$ with any signal or report (whether truthful or not) disobeys the instruction, the maximal instantaneous gain from the deviation is bounded above by $v_{i}(1,1)-R$ and the continuation payoff equals $g^{0}$. On the other hand, the lowest payoff along the path of play equals $\left(1-\delta^{m}\right) \underline{g}+\delta^{m} u$ (by Step 1 ) when subphase $A_{i}$ is just beginning. Hence, (a1) implies that no observable deviation is profitable.

Step 3. No unobservable deviation (in reporting) is profitable.

Since a bidder is made strictly worse off by disobeying the instruction, a profitable deviation is possible only when he misreports his signal and then obeys the instruction. Since the insturctions rules $q^{1}$ and $q^{2}$ are one-shot IC by Lemma 1, bidder $i$ has no incentive to misreport a signal in phases $A_{i}$ and $A_{j}$. Likewise, he has no incentive for misreporting in the punishment phase. It remains to check the incentive for misreporting in phase $S$. 
Let $\pi_{i}\left(s_{i}, \hat{s}_{i}\right)$ denote bidder $i$ 's interim (intertemporal) expected payoff in any period in phase $S$ when he has signal $s_{i}$ and reports $\hat{s}_{i}$ while bidder $j$ reports his signal truthfully.

The discussion below assumes interdependent values. Derivation in the case of private values is similar and hence omitted. We can express $\pi_{i}$ using the continuation payoff $u$ as follows: For $\hat{s}_{i}>r$,

$$
\begin{aligned}
\pi_{i}\left(s_{i}, \hat{s}_{i}\right) & =(1-\delta) \int_{k_{i}\left(\hat{s}_{i}\right)}^{\hat{s}_{i}}\left\{v_{i}(s)-R\right\} f_{j}\left(s_{j} \mid s_{i}\right) d s_{j} \\
& +\delta\left[\int_{0}^{\hat{s}_{i}}\left[x\left(\hat{s}_{i}\right)\left\{\left(1-\delta^{m}\right) \underline{g}+\delta^{m} u\right\}+\left\{1-x\left(\hat{s}_{i}\right)\right\} u\right] f_{j}\left(s_{j} \mid s_{i}\right) d s_{j}\right. \\
& \left.+\int_{\hat{s}_{i}}^{1}\left[x\left(s_{j}\right)\left\{\left(1-\delta^{m}\right) \bar{g}+\delta^{m} u\right\}+\left\{1-x\left(s_{j}\right)\right\} u\right] f_{j}\left(s_{j} \mid s_{i}\right) d s_{j}\right],
\end{aligned}
$$

and for $\hat{s}_{i} \leq r$

$$
\begin{aligned}
\pi_{i}\left(s_{i}, \hat{s}_{i}\right) & =\delta\left[\int_{0}^{\hat{s}_{i}}\left[x\left(\hat{s}_{i}\right)\left\{\left(1-\delta^{m}\right) \underline{g}+\delta^{m} u\right\}+\left\{1-x\left(\hat{s}_{i}\right)\right\} u\right] f_{j}\left(s_{j} \mid s_{i}\right) d s_{j}\right. \\
& \left.+\int_{\hat{s}_{i}}^{1}\left[x\left(s_{j}\right)\left\{\left(1-\delta^{m}\right) \bar{g}+\delta^{m} u\right\}+\left\{1-x\left(s_{j}\right)\right\} u\right] f_{j}\left(s_{j} \mid s_{i}\right) d s_{j}\right] .
\end{aligned}
$$

Upon simplification, we see that (a6) is equivalent to

$$
\begin{aligned}
& \pi_{i}\left(s_{i}, \hat{s}_{i}\right) \\
& =(1-\delta) \int_{k_{i}\left(\hat{s}_{i}\right)}^{\hat{s}_{i}}\left\{v_{i}(s)-R\right\} f_{j}\left(s_{j} \mid s_{i}\right) d s_{j} \\
& +\delta\left[u-\left(1-\delta^{m}\right)(u-\underline{g}) x\left(\hat{s}_{i}\right) F_{j}\left(\hat{s}_{i} \mid s_{i}\right)+\left(1-\delta^{m}\right)(\bar{g}-u) \int_{\hat{s}_{i}}^{1} x\left(s_{j}\right) f_{j}\left(s_{j} \mid s_{i}\right) d s_{j}\right] .
\end{aligned}
$$

Since $k_{i}$ is differentiable almost everywhere, we can differentiate the above with respect to $\hat{s}_{i}$ to obtain

$$
\begin{aligned}
& \frac{\partial \pi_{i}}{\partial \hat{s}_{i}}\left(s_{i}, \hat{s}_{i}\right) \\
& =(1-\delta)\left[\left\{v_{i}\left(s_{i}, \hat{s}_{i}\right)-R\right\} f_{j}\left(\hat{s}_{i} \mid s_{i}\right)-k_{i}^{\prime}\left(\hat{s}_{i}\right)\left\{v_{i}\left(s_{i}, k_{i}\left(\hat{s}_{i}\right)\right)-R\right\} f_{j}\left(k_{i}\left(\hat{s}_{i}\right) \mid s_{i}\right)\right] \\
& -\delta\left(1-\delta^{m}\right)\left[(u-\underline{g})\left\{x^{\prime}\left(\hat{s}_{i}\right) F_{j}\left(\hat{s}_{i} \mid s_{i}\right)+x\left(\hat{s}_{i}\right) f_{j}\left(\hat{s}_{i} \mid s_{i}\right)\right\}+(\bar{g}-u) x\left(\hat{s}_{i}\right) f_{j}\left(\hat{s}_{i} \mid s_{i}\right)\right] \\
& =(1-\delta)\left[\left\{v_{i}\left(s_{i}, \hat{s}_{i}\right)-R\right\} f_{j}\left(\hat{s}_{i} \mid s_{i}\right)-k_{i}^{\prime}\left(\hat{s}_{i}\right)\left\{v_{i}\left(s_{i}, k_{i}\left(\hat{s}_{i}\right)\right)-R\right\} f_{j}\left(k_{i}\left(\hat{s}_{i}\right) \mid s_{i}\right)\right] \\
& -\delta\left(1-\delta^{m}\right)\left[(u-\underline{g}) x^{\prime}\left(\hat{s}_{i}\right) F_{j}\left(\hat{s}_{i} \mid s_{i}\right)+(\bar{g}-\underline{g}) x\left(\hat{s}_{i}\right) f_{j}\left(\hat{s}_{i} \mid s_{i}\right)\right] .
\end{aligned}
$$

On the other hand, $x$ given in (a3) satisfies the following linear differential equation:

$$
x^{\prime}(\alpha)+\frac{\bar{g}-\underline{g}}{u-\underline{g}} z_{j}(\alpha) x(\alpha)= \begin{cases}\frac{1-\delta}{\delta\left(1-\delta^{m}\right)(u-\underline{g})}\left\{v_{i}(\alpha, \alpha)-R\right\} z_{j}(\alpha) & \text { if } \alpha>r, \\ 0 & \text { otherwise. }\end{cases}
$$


(Heuristically, (a3) is derived as the (unique) solution to (a8) with the intial condition $x(r)=0$. (a8) is obtained as follows: The optimality of truth-telling implies the following first-order condition for $s_{i}>r$ :

$$
\begin{aligned}
\frac{\partial \pi_{i}}{\partial \hat{s}_{i}}\left(s_{i}, s_{i}\right) & =(1-\delta)\left[\left\{v_{i}\left(s_{i}, s_{i}\right)-R\right\} f_{j}\left(s_{i} \mid s_{i}\right)-k_{i}^{\prime}\left(s_{i}\right)\left\{v_{i}\left(s_{i}, k_{i}\left(s_{i}\right)\right)-R\right\} f_{j}\left(k_{i}\left(s_{i}\right) \mid s_{i}\right)\right] \\
& -\delta\left(1-\delta^{m}\right)\left[(u-\underline{g}) x^{\prime}\left(s_{i}\right) F_{j}\left(s_{i} \mid s_{i}\right)+(\bar{g}-\underline{g}) x\left(s_{i}\right) f_{j}\left(s_{i} \mid s_{i}\right)\right]=0 .
\end{aligned}
$$

The first line of (a8) follows from the fact that $k^{\prime}\left(s_{i}\right)\left\{v_{i}\left(s_{i}, k_{i}\left(s_{i}\right)\right)-R\right\}=0$ almost everywhere. The similar first-order condition for $s_{i} \leq r$ yields the second line of (a8).)

To verify that truth-telling is (globally) optimal under (a3), we rewrite $\frac{\partial \pi_{i}}{\partial \hat{s}_{i}}\left(s_{i}, \hat{s}_{i}\right)$ using (a8) as follows when $\hat{s}_{i}>r$ :

$$
\begin{aligned}
\frac{\partial \pi_{i}}{\partial \hat{s}_{i}}\left(s_{i}, \hat{s}_{i}\right) & =(1-\delta)\left[\left\{v_{i}\left(s_{i}, \hat{s}_{i}\right)-R\right\} f_{j}\left(\hat{s}_{i} \mid s_{i}\right)-k_{i}^{\prime}\left(\hat{s}_{i}\right)\left\{v_{i}\left(s_{i}, k_{i}\left(\hat{s}_{i}\right)\right)-R\right\} f_{j}\left(k_{i}\left(\hat{s}_{i}\right) \mid s_{i}\right)\right] \\
& +\delta\left(1-\delta^{m}\right)(\bar{g}-\underline{g}) F_{j}\left(\hat{s}_{i} \mid s_{i}\right) x\left(\hat{s}_{i}\right)\left[z_{j}\left(\hat{s}_{i}\right)-\frac{f_{j}\left(\hat{s}_{i} \mid s_{i}\right)}{F_{j}\left(\hat{s}_{i} \mid s_{i}\right)}\right] \\
& -(1-\delta)\left\{\hat{v}_{i}\left(\hat{s}_{i}\right)-R\right\} F_{j}\left(\hat{s}_{i} \mid s_{i}\right) z_{j}\left(\hat{s}_{i}\right) \\
& =(1-\delta) f_{j}\left(\hat{s}_{i} \mid s_{i}\right)\left\{v_{i}\left(s_{i}, \hat{s}_{i}\right)-v_{i}\left(\hat{s}_{i}, \hat{s}_{i}\right)\right\} \\
& +(1-\delta)\left[\frac{f_{j}\left(\hat{s}_{i} \mid s_{i}\right)}{F_{j}\left(\hat{s}_{i} \mid s_{i}\right)}-z_{j}\left(\hat{s}_{i}\right)\right]\left[\hat{v}_{i}\left(\hat{s}_{i}\right)-R-\frac{\delta\left(1-\delta^{m}\right)}{1-\delta}(\bar{g}-\underline{g}) x\left(\hat{s}_{i}\right)\right] F_{j}\left(\hat{s}_{i} \mid s_{i}\right) \\
& -(1-\delta) k_{i}^{\prime}\left(\hat{s}_{i}\right)\left\{v_{i}\left(s_{i}, k_{i}\left(\hat{s}_{i}\right)\right)-R\right\} f_{j}\left(k_{i}\left(\hat{s}_{i}\right) \mid s_{i}\right)
\end{aligned}
$$

Note that it follows from (a3) that

$$
\hat{v}_{i}\left(\hat{s}_{i}\right)-R-\frac{\delta\left(1-\delta^{m}\right)}{1-\delta}(\bar{g}-\underline{g}) x\left(\hat{s}_{i}\right)>0
$$

for any $\hat{s}_{i}>r$. If $\hat{s}_{i}>s_{i}$, then $k_{i}^{\prime}\left(\hat{s}_{i}\right)\left\{v_{i}\left(s_{i}, k_{i}\left(\hat{s}_{i}\right)\right)-R\right\} \geq 0, v_{i}\left(s_{i}, \hat{s}_{i}\right)-v_{i}\left(\hat{s}_{i}, \hat{s}_{i}\right)<0$, and $\frac{f_{j}\left(\hat{s}_{i} \mid s_{i}\right)}{F_{j}\left(\hat{s}_{i} \mid s_{i}\right)}-z_{j}\left(\hat{s}_{i}\right) \leq 0$. Therefore, $\frac{\partial \pi_{i}}{\partial \hat{s}_{i}}\left(s_{i}, \hat{s}_{i}\right) \leq 0$. Similarly, $\frac{\partial \pi_{i}}{\partial \hat{s}_{i}}\left(s_{i}, \hat{s}_{i}\right) \geq 0$ when $\hat{s}_{i} \in\left(r, s_{i}\right)$. When $\hat{s}_{i}<r$, we can similarly show from (a7) and (a8) that $\frac{\partial \pi_{i}}{\partial \hat{s}_{i}}\left(s_{i}, \hat{s}_{i}\right) \leq 0$ (resp. $\geq 0$ ) for $\hat{s}_{i}>s_{i}\left(\right.$ resp. $\left.<s_{i}\right)$. In either case, $\pi_{i}\left(s_{i}, \cdot\right)$ is single-peaked at $\hat{s}_{i}=s_{i}$. This completes the proof of the theorem. //

Proof of Theorem 2: It is clear from the discussion after Theorem 1 that $u^{d}>(\bar{g}+\underline{g}) / 2$. It will be shown below that $u^{d}>g^{0}$. It follows from (a8) that for $\alpha>R$,

$$
x(\alpha)=\frac{1-\delta}{\delta\left(1-\delta^{m}\right)(\bar{g}-\underline{g})}(\alpha-R)-\frac{u-\underline{g}}{(\bar{g}-\underline{g}) z_{j}(\alpha)} x^{\prime}(\alpha) .
$$


Substituting this by noting $g^{*}=\int_{R}^{1}(\alpha-R) F_{i}(\alpha) f_{i}(\alpha) d \alpha$, we have

$$
\begin{aligned}
& \int_{R}^{1} x(\alpha) F_{i}(\alpha) f_{i}(\alpha) d \alpha \\
& =\frac{1-\delta}{\delta\left(1-\delta^{m}\right)(\bar{g}-\underline{g})} g^{*}-\frac{u-\underline{g}}{\bar{g}-\underline{g}} \int_{R}^{1} F_{i}(\alpha)^{2} x^{\prime}(\alpha) d \alpha \\
& =\frac{1-\delta}{\delta\left(1-\delta^{m}\right)(\bar{g}-\underline{g})} g^{*}-\frac{u-\underline{g}}{\bar{g}-\underline{g}}\left[x(1)-2 \int_{R}^{1} x(\alpha) F_{i}(\alpha) f_{i}(\alpha) d \alpha\right],
\end{aligned}
$$

where the second equality follows from integration by parts. It follows that

$$
(\bar{g}+\underline{g}-2 u) \int_{R}^{1} x(\alpha) F_{i}(\alpha) f_{i}(\alpha) d \alpha=\frac{1-\delta}{\delta\left(1-\delta^{m}\right)} g^{*}-(u-\underline{g}) x(1) .
$$

By (a 5$), \varphi(u)$ equals

$$
\begin{aligned}
\varphi(u) & =u-g^{*}+(2 u-\bar{g}-\underline{g}) \frac{\delta\left(1-\delta^{m}\right)}{1-\delta} \int_{R}^{1} x(\alpha) F_{i}(\alpha) f_{i}(\alpha) d \alpha \\
& =u-2 g^{*}+\frac{\delta\left(1-\delta^{m}\right)}{1-\delta}(u-\underline{g}) x(1) .
\end{aligned}
$$

Substituting

$$
x(1)=\frac{1-\delta}{\delta\left(1-\delta^{m}\right)(\bar{g}-\underline{g})} \int_{R}^{1}\left\{1-F_{i}(\alpha)^{\frac{\bar{g}-\underline{g}}{u-\underline{g}}}\right\} d \alpha
$$

from (a3) into (a9), we obtain

$$
\varphi(u)=u-\int_{R}^{1}\left\{1-F_{i}(\alpha)^{2}\right\} d \alpha+\frac{u-\underline{g}}{\bar{g}-\underline{g}} \int_{R}^{1}\left\{1-F_{i}(\alpha)^{\frac{\bar{g}-\underline{g}}{u-\underline{g}}}\right\} d \alpha .
$$

We now show that $\varphi(u)=0$ has a solution in $\left(g^{0}, g^{*}\right)$. It suffices to show that $\varphi\left(g^{0}\right)<0$ since we already know from the remark before Corollary 1 that $\varphi\left(g^{*}\right)>0$. It can be readily verified that in both first-price or second-price sealed-bid auctions, the (symmetric) one-shot Nash equilibrium payoff is given by

$$
g^{0}=\int_{R}^{1}\left\{F_{i}(\alpha)-F_{i}(\alpha)^{2}\right\} d \alpha .
$$

Substituting this into the first term of $\varphi\left(g^{0}\right)$, we get

$$
\varphi\left(g^{0}\right)=-\int_{R}^{1}\left\{1-F_{i}(\alpha)\right\} d \alpha+\frac{g^{0}-\underline{g}}{\bar{g}-\underline{g}} \int_{R}^{1}\left\{1-F_{i}(\alpha)^{\frac{\bar{g}-\underline{g}}{g^{0}-\underline{g}}}\right\} d \alpha .
$$

If we define the function $\phi_{\alpha}(\cdot): \mathbf{R}_{+} \rightarrow \mathbf{R}$ by

$$
\phi_{\alpha}(z)=\frac{1}{z}\left\{1-F_{i}(\alpha)^{z}\right\}
$$


for each $\alpha \in[0,1]$, then it follows from the above that $\varphi\left(g^{0}\right)$ can be rewritten as

$$
\varphi\left(g^{0}\right)=\int_{R}^{1}\left\{\phi_{\alpha}\left(\frac{\bar{g}-\underline{g}}{g^{0}-\underline{g}}\right)-\phi_{\alpha}(1)\right\} d \alpha .
$$

Since $\phi_{\alpha}^{\prime}(z)<0$ for each $\alpha \in[0,1)$ and $z \in \mathbf{R}_{+}, \phi_{\alpha}(z)$ is a strictly decreasing function of $z$ for every $\alpha \in[R, 1)$. It follows that $\varphi\left(g^{0}\right)<0$ since $(\bar{g}-\underline{g}) /\left(g^{0}-\underline{g}\right)>1$. This completes the proof of the theorem. //

\section{References}

Athey, S. and K. Bagwell (1999), "Optimal collusion with private information," mimeo.

Athey, S., K. Bagwell, and C. Sanchirico (1998), "Collusion and price regidity," mimeo.

Baldwin, L. H., R. C. Marshall and J.-F. Richard (1997), "Bidder collusion at forest service timber sales," Journal of Political Economy, 105, pages 657-99.

Brusco, S. and G. Lopomo (2000), "Collusion via signaling in open ascending price auctions with multiple objects and complementarities," mimeo.

Engelbrecht-Wiggans, R. and C. Kahn (1998), "Low revenue equilibria in simultaneous ascending price auctions," mimeo.

Forges, F. (1986), “An approach to communication equilibria," Econometrica, 54, 1375-1385.

Fudenberg, D., D. Levine, and E. Maskin (1994), "The folk theorem with imperfect public information," Econometrica, 62, 997-1039.

Graham, D. A. and R. C. Marshall (1987), "Collusive bidder behavior at single object second price and English auctions," Journal of Political Economy, 95, 1217-1239.

Graham, D. A., R. C. Marshall and J.-F. Richard (1990), "Differential payments within a bidder coalition and the Shapley value," American Economic Review, 80, 493-510.

Hendricks, K. and R. Porter (1989), "Collusion in auctions," Annals d'Economie et de Statistique, 15-16, 217-230.

Johnson, P. and J. Robert (1998), "Collusion in a model of repeated auctions," mimeo.

Mailath, G. and P. Zemsky (1991), "Collusion in second price auctions with heterogeneous bidders," Games and Economic Behavior, 3, 467-486.

Marshall, R. C. and M. J. Meurer (1995), "Should bid rigging always be an antitrust violation," Working paper \#7-95-2, Pennsylvania State University. 
McAfee, R. P. and J. McMillan (1992), "Bidding rings," American Economic Review, 82, $579-599$.

Milgrom, P. R. and R. Weber (1982), "A theory of auctions and competitive bidding," Econometrica, 50, 1059-1122.

Myerson, R. B. (1986), “Multistage games with communication," Econometrica, 54, 323-358.

Robinson, M. S. (1985), "Collusion and the choice of auction," Rand Journal of Economics, 16, 141-145.

Skrzypacz, A. and H. Hopenhayn (1999), "Bidding rings in repeated auctions," mimeo.

von Ungern-Sternberg, T. (1988), "Cartel stability in sealed-bid second-price auctions," Journal of Industrial Economics, 36, 351-358. 


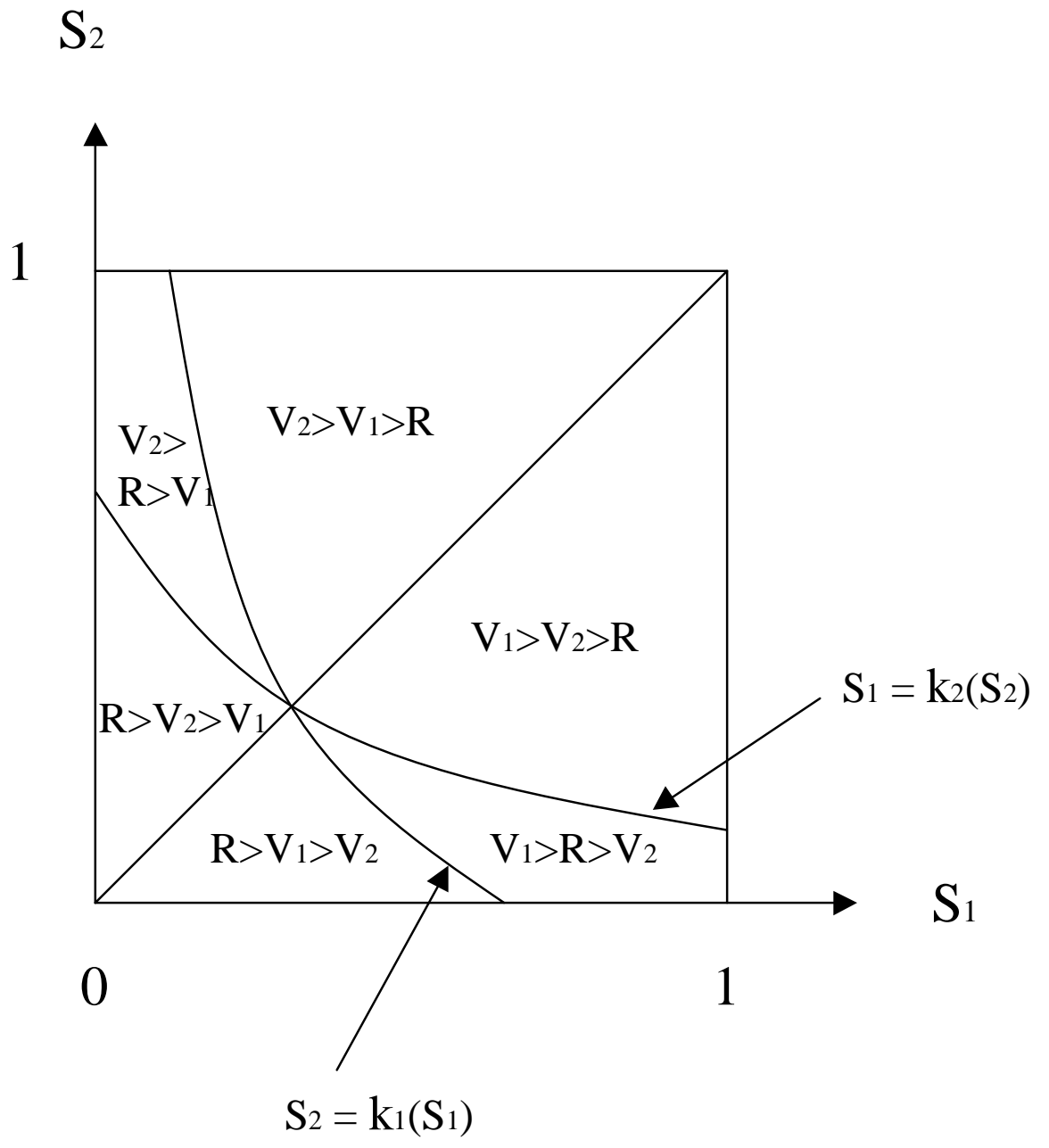

Figure 1 